Indications included intracranial events, head and neck cancers and dysphagia secondary to gastroenterological, neuromuscular or neurodegenerative conditions.

30-day complications: Stoma site infections; (15.8\% for PEG and $19.6 \%$ for RIG), chest infections; $(6.58 \%$ for PEG and $6.55 \%$ for RIG) and minor complications (including blocked or dislodged tube); (5.26\% for PEG and 11.9\% for RIG). Major complications were low (0\% for PEG and $2.97 \%$ RIG - including perforation $(0.60 \%)$, respiratory arrest $(0.60 \%)$, desaturation $(0.60 \%)$ and pneumoperitoneum $(1.19 \%))$. 30-day all-cause mortality was $6.58 \%$ (PEG) and $8.33 \%$ (RIG).

Conclusion Pre-screening PEG referrals identified more inappropriate cases than those referred for RIG. With the exception of chest infections, 30 day minor and major complications were lower in the PEG group, as was 30-day all-cause mortality. We hypothesise that the less rigorous screening process may be contributing to excess complication and mortality rates of RIG insertion. This may, or may not be unique to our Trust. RIG is usually the second line method of insertion, and we recognise that this patient group may have a poorer premorbid state. We recommend formal assessment of all gastrostomy referrals regardless of insertion technique. The Nutrition Team is currently looking towards pre-screening all gastrostomy referrals.

Disclosure of Interest None Declared.

\section{PTH-133 ELECTRONIC PERSONAL HEALTH RECORDS FOR PATIENTS ON HOME PARENTERAL NUTRITION: A PATIENT SATISFACTION SURVEY}

${ }^{1} \mathrm{~T}$ Ambrose*, ${ }^{2} \mathrm{R}$ Topan, ${ }^{1} \mathrm{M}$ Small, ${ }^{1} \mathrm{JM}$ Nightingale, ${ }^{1} \mathrm{SM}$ Gabe. 'Lennard Jones Intestinal Failure Unit, St Mark's Hospitall, London, UK; ${ }^{2}$ Northwick Park Hospital, London, UK

\subsection{6/gutjnl-2014-307263.579}

Introduction The public demand for flexible access to health information and services is growing, encouraged by Intnet trends and policies promoting patient involvement. Patients Know Best ${ }^{\circledR}(\mathrm{PKB})$ is an electronic patient centred system providing a secure forum for patients to interact with healthcare teams. With increasing use of this system by our home parenteral nutrition patients we aimed to assess patient satisfaction with a survey.

Methods PKB was introduced to patients during routine clinic visits and verbal consent obtained. We recorded the frequency of use, total number of electronic discussions held and the number of additional carers (other healthcare staff or family members) involved in their personal health record. We distributed a 10 question survey to all users.

Results 119 patients (50 male, 69 female) were registered over a period of 18 months with a median age of 49 years (range 1785 years, mean 48 years). A total of 5015 unique electronic conversations were recorded. These would usually have occurred via telephone. PKB has been used for 4 patients transitioning from paediatric to adult services and 2 patients from abroad. Other patients invited included 128 outside clinicians (eg local nutrition nurses and dietitians, transplant coordinators) and 29 carers. There were 58/119 (48.7\%) responses including 1 incomplete dataset (61\% female). $31 / 57$ patients $(54.4 \%)$ were over 50 years of age. $42 / 57(73.7 \%)$ received parenteral nutrition and 13/57 $(22.8 \%)$ fluids and electrolytes. 51/58 (87.9\%) patients felt at least "somewhat confident" working online with their healthcare team and the same number felt that having access to the results was at least "somewhat helpful" - 32 (62.7\%) of these responding "very/extremely helpful". 30/58 (51.7\%) use PKB a few times a month, $4 / 58(6.9 \%)$ a few times a week and the remainder less frequently. The more useful features of PKB included Discussions (ie contacting the St Mark's Nutrition team electronically) and Monitoring (ie test results). Patients use PKB to contact doctors/nutrition nurses more than dietitians/ administrators.

Conclusion Our survey suggests that our patient cohort find this a useful facility to contact us and improve the management of their long-term conditions. Not all patients access PKB regularly and they only had a short period in which to respond to the survey. This may explain the $49 \%$ response rate in an otherwise motivated patient population. This is an emerging and effective way for patients to interact with their healthcare teams and we have not found it has increased our workload. The ability to communicate seamlessly between different healthcare professionals reduces the current difficulties which exist when transferring information between multiple care providers (eg HIFNET). Disclosure of Interest None Declared.

\section{PTH-134 MALNUTRITION SCREENING IN HOSPITAL OUTPATIENTS}

${ }^{1} \mathrm{Z}$ Gundkalli, ${ }^{2} \mathrm{~V}$ Blackwell, ${ }^{2} \mathrm{~W}$ Howson*, ${ }^{2} \mathrm{H}$ Williams. 'Imperial College School of Medicine, London, UK; ${ }^{2}$ Imperial College Healthcare NHS Trust, London, UK

\subsection{6/gutjnl-2014-307263.580}

Introduction NICE Guidelines (2006) advocate the screening of all new hospital outpatients in order to identify individuals at risk of malnutrition within the community. However, existing data in support of outpatient screening are limited. To establish the utility of screening in specific outpatient settings, data were collected from targeted clinics. Patterns of risk associated with age, gender, ethnicity and 'new' or 'follow up' outpatient status were determined.

Methods The Malnutrition Universal Screening Tool (MUST) was used to assess the nutritional status of all individuals attending outpatient appointments at eight clinics at St. Mary's Hospital, Imperial College Healthcare NHS Trust. These were Gastroenterology, General Surgery, Geriatric Falls, Oncology, Chest and Allergy, Hypertension, Dermatology and Gynaecology/Urogynaecology. Appropriate action was taken as per guidelines for patients identified as 'at-risk'.

Data were analysed using descriptive statistics, chi-square and logistic regression.

Results 585 outpatients were screened over a six week period (male 35.9\%, $\mathrm{n}=210 / 585$; female 64.3\%, $\mathrm{n}=375 / 585$; median age $=52$, range $16-91$ years; white ethnicity $57.7 \%$, $\mathrm{n}$ $=338 / 585$; non-white ethnicity 42.3\%, $\mathrm{n}=247 / 585)$. The overall prevalence of malnutrition risk was $12.1 \%(\mathrm{n}=71 / 585)$. There was a significantly higher risk of malnutrition in the young (16-24 years) and the elderly ( $\geq 75$ years) $(p=0.04)$ and in individuals of white ethnicity $(\mathrm{p}=0.0002)$. There was no difference in the prevalence of risk between new or follow up patients.

There was a predictably high yield in Gastroenterology, General Surgery, Oncology and Geriatric Falls clinics. Low prevalence of risk was found in Hypertension and Gynaecology/ Urogynaecology clinics.

The Dermatology clinic yielded notable results with a $15.0 \%$ ( $\mathrm{n}=12 / 80$ ) prevalence of risk. 8/12 of these patients were identified as medium risk (MUST score 1) due to a BMI of 18.5$20.0 \mathrm{~kg} / \mathrm{m}^{2} ; 7 / 8$ were female and of white ethnicity.

Conclusion There have been no published studies of larger cohorts in a hospital outpatient setting. 